\title{
EL PALEOLÍTICO EN CIUDAD REAL. SÍNTESIS VALORATIVA
}

\author{
THE PALAEOLITHIC IN CIUDAD REAL. SYNTHESIS
}

por

ANTONIO CIUDAD SERRANO

El comienzo de un poblamiento inicial, que sirve de partida al devenir histórico provincial, es una herencia que hunde sus raíces en las remotas etapas del Paleolítico. En el estudio de los orígenes y primer desarrollo de este poblamiento podemos distinguir: primero, un poblamiento paleolítico inicial en el achelense inferior arcaico, con industrias de cantos tallados evolucionados, para continuar, en un segundo momento, con una ocupación progresiva durante el achelense medio y superior, y asistir, en un tercero, a una amplia difusión con los complejos musterienses durante el Paleolítico Medio. Finaliza este primer poblamiento con su desaparición y el vacío del Paleolítico Superior, fenómeno no exclusivo de nuestra provincia sino que afecta también con despoblamientos comarcales a otras latitudes en la nueva redistribución humana de esta etapa.

\section{EL MEDIO FÍSICO}

Teniendo en cuenta la incuestionable y condicionante conexión del medio físico con las culturas, creemos lógico y conveniente detenernos en la consideración de algunos aspectos de este medio provincial.

\section{A) Red hidrográfica}

Iniciamos la síntesis del medio físico con unos apuntes resumidos de la red hidrográfica ya que los depósitos más recientes de la superficie provincial, sitios ocupacionales en el Paleolítico, se originaron por la acción de esta red cuaternaria al encajarse en la superficie pliocena.

Hidrográficamente, la provincia de Ciudad Real se encuentra atravesada por ríos y afluentes de dos grandes sistemas hidrográficos pertenecientes, respectivamente a dos de las grandes cuencas españolas: la del Guadiana y la del Guadalquivir.

El Guadiana atraviesa la provincia de E. a W. y su curso suele dividirse, para su mejor estudio y conocimiento, en Guadiana Alto o de las Lagunas de Ruidera, Guadiana de la Mancha y Guadiana de los Montes de Toledo. 
El primero, a los pocos kilómetros de su nacimiento, en la zona limítrofe de Albacete con Ciudad Real, atraviesa una serie de lagunas (Lagunas de Ruidera), situadas a diferentes alturas, y tras recorrerlas adquiere un mayor caudal, entrando en el estrecho de Peñarroya al pantano actual del mismo nombre. El segundo, Guadiana de la Mancha, encamina sus aguas a la llanura manchega frente a Argamasilla de Alba, recorriendo una amplia red de abanicos aluviales; tras su paso por esta zona de abanicos aluviales, las aguas en su marcha S-N atraviesan una fase carbonatada, génesis de karstificación; en esta zona se desarrolla el sistema fluvial de los subsidiarios, Záncara, en el que se distinguen cuatro niveles de terrazas, y Gigüela que impulsa en su confluencia con el Guadiana una fuerza mayor en éste, entrando en una llamada “Zona de Transición”', con aparición de restos de terrazas y en la que se le une el río Bañuelos, con el Becea, existiendo en ellos hasta tres niveles de terrazas. El Guadiana de los Montes de Toledo se inicia en el Puente de Alarcos, penetrando en las estribaciones más meridionales de estos montes; por Corral de Calatrava, recibe las aguas del Jabalón que, originado en el Campo de Montiel, surca el Campo de Calatrava y en el que se distinguen un claro sistema de terrazas; en las inmediaciones de Luciana, desemboca el Bullaque, procedente de los Montes de Toledo, y en cuya cuenca fluvial pueden descubrirse una serie de abanicos aluviales y hasta tres niveles de terrazas (REDONDO y MOLINA 1980); en su trayecto hasta Puebla de D. Rodrigo se observan, en un primer tramo, los escalonamientos producidos por la raña y por los niveles de terraza y, en un segundo, se marcan aún más estos niveles; en estos parajes recibe las aguas de algunos afluentes, e. g. Río Frío y Tirteafuera, subcuencas de interesantes hallazgos.

El segundo sistema hidrográfico provincial pertenece al Guadalquivir, con una extensión drenada mucho menor, desarrollada en la parte más meridional de la provincia.

Como afluentes principales, únicos de la red que recogen las aguas, destacamos los ríos Guadalmena, Guadailén y el Jándula que recibe las aguas del Robledillo, Ojailén, Fresnedas y Montoro, cursos también de importantes localizaciones.

Junto con la red hidrográfica, mención importante merecen las lagunas, frecuentes en el Campo de Calatrava. Muchas de ellas deben su origen a explosiones volcánicas y consisten en un cráter cuyo fondo suele situarse por debajo del nivel del suelo. Sus inmediaciones han sido muy atractivas en algunos períodos para el hombre paleolítico y en sus alrededores se dan numerosos asentamientos.

\section{B) Estratigrafía geológica}

Los yacimientos paleolíticos superficiales se encuentran sobre materiales de diverso tipo generalmente cuaternarios y, salvo uno donde se encontró industria “in situ”, en el depósito no se puede asimilar la edad del yacimiento directamente ya que los instrumentos trabajados lo han sido sobre cantos de cuarcita ya depositados, relacionados con un suelo muchas veces de color rojizo bastante arcilloso. Y es en relación a este suelo con el que estarían "in situ", ya que se ve en casi todos, cómo las labores de arado de los campos van sacando estos materiales a la superficie.

Consecuentemente, la datación de la industria podría hacerse en correlación al periodo de formación del suelo o a un momento anterior.

Los suelos más típicos son los paleosuelos rojos muy frecuentes en el Campo de Calatrava. MONTURIOL determina dos momentos a lo largo del Cuaternario en los que se formaron suelos rojos bajo un clima cálido y lluvioso, correspondiendo el primero de ellos posiblemente al Mindel y el segundo al Würm I.

En cuanto a los tipos de depósitos sobre los que se desarrollan estos suelos, tenemos materiales cuaternarios tales como depósitos de abanico aluvial, de terraza, de ladera y material volcánico.

Los abanicos aluviales, que tienen una gran importancia y desarrollo en algunas zonas (tales como en los límites del Campo de Montiel o en la zona de los Montes de Toledo), se puede atribuir su formación a una fase tectónica finipliocena en la que por reactivación de fallas se produjo un levantamiento de bloques. 
Los abanicos aluviales son los que con más frecuencia sirven de soporte a los yacimientos y, como más destacables, pueden citarse los de la zona de Porzuna y aledaños en donde a una depresión central vienen a coalecer los abanicos procedentes de las sierras cuarcíticas paleozóicas del Norte y del Sur.

En cuanto a las terrazas, se han encontrado una serie de niveles de terraza en el río Guadiana y sus afluentes, Bañuelos y Jabalón, y una correlación entre ellos de las tres terrazas inferiores, siendo difícil hacerlo para las superiores por insuficiencia de datos.

Los depósitos de ladera están muy desarrollados al pie de las sierras cuarcíticas de edad paleozóica. Para estos depósitos de piedemonte se piensa en un posible origen poligénico con la intervención de una fase crioclástica durante un periodo frío en el que se dieron condiciones periglaciares que liberarían gran cantidad de cantos irregulares y heterométricos, material clástico que durante los periodos pluviales se distribuirían por la ladera.

Estas condiciones de periodos fríos, necesarios para que se pueda dar una fragmentación mediante la acción del hielo en las grietas que afectan a las rocas, se debieron dar en los momentos glaciares cuaternarios. Pero tal como algunos indican, e. g. CRUSAFONT, la Península Ibérica debido a su baja latitud debió, durante estas épocas glaciares, estar más cerca de las condiciones que reinaron en el dominio pluvial africano que las que existieron en el boreal europeo. Las dos únicas glaciaciones, que como tal afectaron a la Península, fueron la Riss y Würm y, de ellas, solo el Würm pudo afectar plenamente a toda la superficie del territorio, manifestándose de forma diversa en la parte septentrional y meridional; en una reinarían condiciones glaciares y en otra, periglaciares. Es muy fácil que estos piedemontes tan frecuentes y bien desarrollados en la base de las sierras se hayan formado en estos períodos periglaciares, especialmente durante el Würm, con una manifestación más intensa.

Los depósitos volcánicos que se encuentran extraídos en la zona del Campo de Calatrava han sido estudiados de una forma precisa por ANCOECHEA. La edad de las manifestaciones volcánicas no nos serviría para una acotación más precisa de la posible edad de los yacimientos paleolíticos ya que el más reciente de los volcanes es anterior al Mindeliense y la utilización de la materia prima volcánica es muy circunstancial y esporádica.

Con todos los datos disponibles, tanto geológicos como los referidos a los yacimientos en su medio físico, se puede estructurar la siguiente secuencia estratigráfica geológica en el ámbito provincial y su entronque con el Paleolítico Inferior y Medio.

\section{PALEOLÍTICO INFERIOR:}

\section{Achelense antiguo:}

Terrazas de $+19 / 20$ m y 30 del Jabalón

Depósito de $25 \mathrm{~m}$ por encima del Guadiana (tramo central)

Terraza de $+6 \mathrm{~m}$ del Alto Guadiana

Abanico aluvial en el Campo de Calatrava correlacionado con la terraza de + 30 m del Jabalón.

\section{Achelense Medio:}

Antiguo: Terraza de $+14 \mathrm{~m}$ del Guadiana (tramo central)

Reciente: Terraza de $+6 \mathrm{~m}$ del Guadiana (tramo central)

Terraza de +7 m del Jabalón.

\section{Achelense Superior:}

Formaciones de suelos rojos sobre la terraza de $+6 \mathrm{~m}$ y abanicos aluviales correlacionados. 


\section{PALEOLÍTICO MEDIO:}

\section{Terraza de $+2 / 3 \mathrm{~m}$ del Guadiana}

Piedemontes sobre la terraza de $+6 \mathrm{~m}$ del Guadiana y +10 del Jabalón y suelos sobre la terraza de $11 / 13 \mathrm{~m}$ del Bullaque en el umbral situado al NO. de Piedrabuena.

Suelos rojos sobre abanicos aluviales para yacimientos en posición dominante

Bordes de antiguas o aún actuales lagunas

\section{C) Fauna}

Para el conocimiento de la fauna provincial, algunos hallazgos son orientativos de las especies faunísticas durante el Pleistoceno inferior.

En relación con materiales volcánicos, está el hallazgo en Valverde (Hernández Pacheco 1921) de restos fósiles, datables en la época indicada (Aguirre Enríquez 1971).

El yacimiento paleontológico donde se han localizado se encuentra aproximadamente a kilómetro y medio al E. de Alcolea de Calatrava, y a menos de un kilómetro de la actual margen del Guadiana. Los restos de fauna fósil localizados pertenecen a cuatro especies de mamíferos: Elephas meridionalis Nesti, Hippopotamus amphibius Linné, subespecie major Owen, Equus caballus fossilis y Cervus.

Del Elephas meridionalis se rescató un molar superior y un gran fragmento de otro en el que se conservaba la mayor parte de la corona.

Los restos del Hippopotamus amphibius, subespecie major, por la longitud de su cráneo calculada en un metro, eran un fragmento de canino inferior izquierdo de unos $25 \mathrm{~cm}$ de largo; otro fragmento de unos $5 \mathrm{~cm}$ de un canino inferior derecho; un fragmento del último molar izquierdo; y un fragmento de cráneo correspondiente a la porción orbitaria.

Del Equus caballus fossilis, aparecieron dos molares, uno inferior con la corona en excelente estado de conservación y desgaste, y otro superior desgastado por el uso.

Correspondientes a un Cervus, son un fragmento de mandíbula izquierda con el último molar y un molar suelto de esta misma mandíbula.

También del Pleistoceno Inferior, son los restos estudiados por Aguirre Enríquez, procedentes de unas explotaciones de manganeso, actualmente cerradas, cercanas al Castillo de Calatrava la Vieja, y compuestos por una muela última, muy fragmentada, de un Mammuthus meridionalis, incluyendo en esta etapa, además, varios huesos de un Bovino, con toda probabilidad un Leptobos.

$\mathrm{Si}$ bien correspondientes estos restos a la etapa concreta de un Pleistoceno Inferior, de los otros momentos pleistocénicos no tenemos datos, podemos apreciar como la macrofauna provincial presenta una variedad de especies que podríamos considerar como básicas por su presencia constante durante todo el Paleolítico ibérico, caballo, toro, etc., a los que se unieron otras especies reveladoras de los cambios climáticos.

\section{LOS DATOS ARQUEOLÓGICOS DEL PALEOLÍTICO INFERIOR Y MEDIO}

Los datos arqueológicos publicados del Paleolítico Inferior y Medio en Ciudad Real, abarcan más de cien yacimientos. La ordenación seriada de estas abundantes localizaciones vertebran, sin solución de continuidad, la siguiente secuencia arqueológica:

\section{PALEOLÍTICO INFERIOR:}

\section{Achelense inferior arcaico evolucionado}




\section{Achelense medio:}

Antiguo

Evolucionado

Achelense superior:

Inicial

Final con micoquiense

\section{PALEOLÍTICO MEDIO-MUSTERIENSE:}

Facies Tipo Quina

Facies Levallois

\section{A) Achelense inferior arcaico evolucionado.}

A esta etapa corresponden los hallazgos del Molino del Emperador, Terraza de $+19 / 20$ m en la orilla derecha del río Jabalón y Terraza de +30 m aguas arriba de Puente Morena, localizaciones efectuadas por M. Santonja, más los yacimientos, abundantes en material de Santa María del Guadiana, Laguna Blanca y Arenales de Turra (en Argamasilla de Alba, Argamasilla de Calatrava y Villahermosa, respectivamente).

A estos, y como probables, pueden unirse los El Carnerín (Corral de Calatrava), Las Eras y los Areneros, (Argamasilla de Alba), Puente Picón (Picón) y Valverde.

El soporte geológico de los atribuidos como ciertos a este Achelense inferior arcaico evolucionado comprende las terrazas de + 19/20 m y 30 m del Jabalón, la de + 6 m del Alto Guadiana y un abanico aluvial en el Campo de Calatrava correlacionado con la terraza de + $30 \mathrm{~m}$ del Jabalón y con la primera formación de los suelos rojos bajo un clima cálido y lluvioso, con toda probabilidad durante la glaciación Mindel (Monturiol et alii 1970).

Tecnológica y tipológicamente, las industrias de estos yacimientos revelan un claro arcaísmo, tanto en los restos de talla (núcleos y lascas), como en el utillaje instrumental. Centrando nuestra atención en estos últimos, podemos apreciar que la base industrial está compuesta por cantos tallados, con mayor presencia de los tallados bifacialmente que los de talla unifacial. Junto a estos instrumentos que dominan masivamente estas industrias, existe la presencia significativa de bifaces de técnica primitiva la mayor parte de ellos; existe, además, un inicio de diversificación de piezas tipológicas, e.g. hendedores, triedros, raederas, etc., cuyo peso especifico en el conjunto total no es determinante pero son exponentes de un proceso evolutivo técnico y tipológico a las etapas culturales del Achelense Medio.

\section{B) Achelense Medio}

El Achelense medio queda centrado geográficamente en el foco del Campo de Calatrava, con las localizaciones de El Martinete, Puente Pozuelos y Albalá, en la cuenca del Guadiana, y en el Campo de Montiel por el del Camino del Picayuelo en el nacimiento del río Villanueva, afluente del Guadalquivir.

Las formaciones geológicas de las industrias del Achelense medio son: Medio antiguo, terraza de $+14 \mathrm{~m} \mathrm{del}$ Guadiana (tramo central); Medio reciente, terraza de $+6 \mathrm{~m}$ del Guadiana (tramo central) y terraza de $+7 \mathrm{~m}$ del Jabalón.

Las industrias de estos yacimientos, con un avance técnico y tipológico básico respecto a las anteriores, abarcan desde un estadio del Achelense medio antiguo, representado por El Martinete y Puente Pozuelos, 
hasta horizontes culturales evolucionados de un Achelense medio reciente y de transición al Superior, a tenor de las series líticas de Albalá y las más antiguas del Camino del Picayuelo.

Los instrumentos más representativos de sus respectivas industrias son los bifaces ya clásicos, con un destacado predominio de los amigdaloides, más de la mitad, con escasa representación de formas más avanzadas, lanceoladas, cordiformes u ovaladas. Es destacable en estas industrias el importante número de triedros, casi al mismo nivel porcentual que los bifaces, junto con otro rasgo técnico digno de tener en cuenta, el de los abundantes hendedores que, como en el caso de los bifaces, las formas más antiguas, al parecer la 0 y la II, son las que más se dan.

\section{C) Achelense superior}

Durante el Achelense superior, y reflejando sin duda una densificación del poblamiento, nos encontramos con un elevado número de localizaciones. Se origina una redistribución de los grupos humanos que se asientan a las orillas de los afluentes del Guadiana, más que en éste mismo, ocupando también zonas de encharcamiento.

Junto a esta penetración territorial, se amplía, acompasado al avance de la tecnología, el aprovechamiento de los recursos de materia prima para la talla, sustituyendo los guijarros redondeados y sin aristas por los cantos angulosos, con utilización de otras piedras locales, e.g. areniscas o rocas volcánicas.

Los yacimientos de este Achelense superior se distribuyen desde el Alto Guadiana, a través de todo su recorrido por la provincia, extendiéndose por sus afluentes, Jabalón y Tirteafuera por su izquierda y Bañuelos y Bullaque por la derecha, y apareciendo también en zonas endorréicas interiores del Campo de Calatrava en donde forman un denso foco.

Todo el acervo de datos publicados es fiel exponente de este Achelense superior cuyo horizonte industrial más completo está representado por las series de Porzuna, razón por la que nos detenemos en él de forma particular.

\section{El yacimiento de Porzuna}

Desde su descubrimiento en 1974, el área de los hallazgos de Porzuna, en torno a las aldeas de este ayuntamiento, Las Tiñosillas y Las Casas del Río, en ambas márgenes del Bullaque, ha sido sometida a una intensa labor prospectora. La ingente cantidad de materiales se reunió en varias colecciones particulares, siendo las más numerosas las de D. Estanislao Oliver Cabezas, estudiada y publicada en 1979 (Vallespí; Ciudad y G ${ }^{a}$. Serrano 1979), y las de D. Alfonso Retamosa, padre e hijo, y D. Margarito Expósito Rodríguez, de las que se estudiaron sendas amplias muestras que previamente ellos habían ido seleccionando, (Vallespí; Ciudad y Ga. Serrano 1985).

El área de recogida ocupa una gran extensión y el cúmulo de materiales analizados se atribuyen a industrias del Paleolítico Inferior y Medio, substancialmente del Achelense superior y Musteriense, atestiguando con claridad que se trata de un complejo arqueológico de concentración en el área explorada de varias localizaciones distintas, de las que ahora nos interesan las del Achelense superior. El yacimiento debe interpretarse como una concentración local de la red de ocupación de la cuenca del Bullaque, al amparo del área comarcal de sus recursos durante los tiempos del último interglaciar hasta mediados de la glaciación Würm. Este amplio espacio se convirtió en la cantera de un vasto taller de explotación, in situ, de la materia prima local; la acumulación, junto a los innumerables desechos de talla, de series no menos abundantes de utillaje apunta a la consideración del lugar como asentamientos duraderos, de sitios de actividades, cazaderos con áreas de despedazado, llevándonos a la presunción del apoyo consiguiente de unos hábitats, si bien de estos últimos aspectos faltan de momento restos indicativos. 
Aunque no establecida la conexión geológica de las industrias de superficie con las formaciones cuaternarias del Bullaque, el marco geomorfológico local de la cuenca (Redondo y Molina 1980) está constituido por una sucesión de cuatro depresiones estructurales: las de El Molinillo y Retuerta, en su curso alto, la central de El Robledo y, en su bajo curso, la de Piedrabuena, rellenas por materiales detríticos y comunicadas entre sí por una serie de pasos y gargantas; estos umbrales dificultan el drenaje general de la cuenca, produciendo encharcamientos. Dichos depósitos detríticos son de cuatro tipos fundamentales: coraza ferralítica marginal, en los bordes paleozóicos, arenas y arcillas arenosas en la depresión de El Robledo y a lo largo de la carretera de Alcoba a Piedrabuena, al Oeste del Bullaque, depósitos de abanicos aluviales y depósitos de terrazas, que en la depresión de El Robledo forman enormes extensiones planas, donde en algunos casos se localizan hasta tres niveles de terrazas por encima de la línea de inundación actual, cuyos depósitos han recogido directamente de los glacis y abanicos aluviales de la cuenca los abundantes materiales, cantos semirrodados y bloques, que constituyen la materia prima de la industria lítica. Como se trata, según dichos autores, de un marco morfológico en gran parte inalterado desde la instalación de la red fluvial actual, salvo pequeños retoques, su contemplación puede aproximarnos inicialmente a la ambientación del escenario de los asentamientos paleolíticos del lugar.

Desde el punto de vista industrial, el Achelense superior de Porzuna se caracteriza, caracterización extensible a todos los yacimientos coetáneos, por una gran riqueza y variedad de bifaces y hendedores, cuyas formas primitivas quedan muy suavizadas por la presencia de otras más avanzadas; existen también en cantidad menor, pero muy característicos, triedros así como cantos tallados, bolas y esferoides asociables, completándose este cuadro definidor con buenas series de raederas, cuchillos, muescas, etc.

\section{D) Musteriense}

Los yacimientos conocidos del Paleolítico Medio superan el número total de localizaciones anteriores, distribuyéndose por una más amplia extensión territorial y expandiéndose aún más por las redes de afluentes, con penetración por arroyos secundarios, y una significativa elección de rebordes de lagunas y zonas endorréicas, la mayoría antiguas láminas de agua hoy desecadas.

La abundancia de yacimientos y su gran expansión por todo el territorio deben relacionarse con la naturaleza de los lugares de ocupación y la dinámica de sus grupos, pero son índice también de un aumento de la población.

Respecto a su caracterización, el Musteriense provincial ha sido definido como un Musteriense de tradición achelense, a tenor de los datos estudiados sobre todo en el conjunto separado de La Solana de los Monteros, en el yacimiento de Porzuna (Vallespí; Ciudad y Ga . Serrano 1985). Esta calificación, que está relacionada con la tecnología y la tipología, creemos que se ha de interpretar, con la generalización del poblamiento, como culminación del proceso emprendido durante el Achelense superior, continuidad que nos parece confirmada por los muchos lugares de la anterior ocupación con permanencia musteriense y por las mismas industrias líticas musterienses, cuya similitud con las achelenses nos hacen concebir éstas como un fondo común generalizado a partir del cual se desarrollaron las segundas.

Centrando nuestra atención en el referido conjunto de La Solana de los Monteros, que se ha podido aislar del área general de Porzuna, se caracteriza, con extensión genérica al resto de los yacimientos del Paleolítico Medio, su clara naturaleza musteriense por la definición de sus desechos de talla, acompañados por un utillaje con predominio absoluto de las raederas; pero, junto a esta realidad musteriense, existen tipos instrumentales característicos de la anterior etapa achelense, e.g. bifaces y hendedores, de tamaños muy reducidos y formas avanzadas. 
Sobre este Musteriense de tradición achelense peculiar, fondo común de la región, parece que incidieron, si no todas, si algunas modalidades o facies de la clásica tradición de corte francés que si no determinan el que pueda hablarse de facies estrictas, sí se han distinguido y agrupado las industrias en dos corrientes: la primera, tipo Quina, y la segunda, Levallois.

\section{VALORACIÓN COMO PRIMER POBLAMIENTO DE LA REGIÓN}

Nos encontramos, con toda la abundancia de localizaciones, con un amplio número de yacimientos del Paleolítico Inferior y Medio en el ámbito de la provincia. Vertebrados en sus correspondientes series regionales de la Submeseta Sur, suponen un estimable despliegue de hallazgos suficiente, a nuestro modo de ver, para evidenciar el alcance del desarrollo del primer poblamiento humano del territorio. Este poblamiento se iniciaría en el Achelense Inferior arcaico, se desarrollaría durante el Achelense Medio y Superior y alcanzaría su apogeo en el Musteriense, para convertirse luego toda la geografía provincial en una zona deshabitada durante el Paleolítico Superior hasta los momentos de un Calcolítico temprano.

Se ha comprobado que geográficamente las localizaciones se dispersan por todo el territorio provincial, a excepción de la Zona de la Mancha, afectada solo en sus rebordes limítrofes. Los ríos que cruzan la parte de Ciudad Real que es la Llanura Manchega, Azuer, Gigüela y su afluente el Záncara no han proporcionado, hasta el momento, asentamientos paleolíticos. La razón, creemos, debe buscarse en que esta zona está desabastecida de materia prima, cantos rodados como base de las industrias. Al mismo tiempo, al ser una zona escasa en cursos fluviales, aunque solo fueran arroyos afluentes, es otro de los factores negativos en el desarrollo del poblamiento humano paleolítico.

En cuanto a las estructuras geomorfológicas, creemos distinguir una trayectoria en el asentamiento y expansión durante el Paleolítico Inferior. Los yacimientos del Achelense Inferior arcaico se sitúan: uno en terraza de $+6 \mathrm{~m}$ del Guadiana Alto (correspondiente alas altas en el tramo medio); otro en un abanico aluvial, asimilable y cercano a una terraza del Jabalón, donde Santonja recogió algunos testimonios también de esta época; otro en un depósito del Guadiana a una altura de + $25 \mathrm{~m}$; y, finalmente, otro en una formación indeterminada en un arroyo cercano al río Villanueva, afluente del Guadalquivir a través del Guadalmena. En resumen, durante esta etapa, el hombre parece elegir la cercanía de los ríos en terrazas coetáneas y en zonas que podríamos calificar de periféricas o tendentes a la periferia.

Durante el Achelense Medio, las localizaciones siguen realizándose sobre terrazas más bajas que las anteriores y sobre un abanico aluvial. Los ríos sobre los que aparecen son el Guadiana y un afluente de cierta importancia, al menos provincial, del Guadalquivir.

En el Achelense Superior, continúan los asentamientos sobre terrazas, cobran importancia los abanicos aluviales y aparecen yacimientos en zonas endorréicas. Los ríos afluentes cobran y alcanzan mayor importancia, en cuanto a hallazgos, que el propio Guadiana. Podríamos definir este momento como el primero de gran difusión y penetración hacia todas las zonas que denominaríamos habitables por recursos materiales.

El Paleolítico Medio supondría el momento cumbre de la difusión. Terrazas bajas de los ríos, colector y afluentes, arroyos subsidiarios, lagunas y zonas endorréicas, lugares de encharcamiento proporcionan, donde es posible localizar provisión de materia prima, huellas de la presencia humana. Es de notar que el único yacimiento, localizado sobre piedemonte, también corresponde a esta etapa.

En cuanto a las áreas de asentamientos, corresponden, donde se han verificado localizaciones, a formaciones de relieve cuaternarias. Las serranías, elevaciones menores no cultivables actualmente no han proporcionado hallazgos, hecho que se relaciona indudablemente con las afloraciones cuarcíticas de muy mala calidad como materia prima. 
Hay, pues, una vinculación total con los lugares de cantos rodados y angulosos, y observamos además que la vinculación con los primeros se realiza durante el Achelense Inferior arcaico de una forma total y que, sobre todo a partir del Achelense Superior, comienza a diversificarse, uniéndose la elaboración industrial más a los cantos angulosos que a los cantos rodados. Incluso en lugares con series diferenciadas, parece que puede establecerse una distinción de las áreas de cantos para una u otra serie industrial.

Sobre la naturaleza de los yacimientos, parece observarse que la primera etapa se corresponde con talleres permanentes, con áreas geográficas algo amplias y con gran acumulación de cantos rodados, donde el suelo prácticamente está recubierto por los guijarros. Después, durante el Achelense Superior y, sobre todo, en el Paleolítico Medio parece que la envergadura de los yacimientos disminuye (envergadura que se extiende a una no tan intensa acumulación de materia prima en el lugar como a la cantidad de industria), no obstante el caso, al parecer excepcional de Porzuna, que por otra parte ha sido objeto y prolongada prospección durante muchos años. Todo esto estaría, sin duda, en conexión con los géneros de vida, de mayor o menor movilidad relacionada con los modos de caza, y con las técnicas de aprovechamiento de la materia prima que, en el Paleolítico Medio, permiten que cualquier cantorral pueda convertirse en lugar de asentamiento, aunque solo sea pasajero.

Como hemos podido comprobar, las vías de penetración, difusión y asentamiento son vías naturales en relación con los cursos de ríos, arroyos, lagunas o zonas de encharcamiento más temporal. Sobre la cuestión del momento inicial del poblamiento, creemos que hay que relacionarlo con zonas meridionales, de Andalucía. En efecto, los primeros asentamientos están situados, uno muy cercano a un afluente del Guadalmena, que vierte sus aguas en el Guadalquivir; otro en el Alto Guadiana, cerca de las Lagunas de Ruidera, no muy lejano a la zona drenada por el Guadalquivir, y ambos no relativamente distantes; el tercero, en el Campo de Calatrava, en una zona limítrofe del mismo, bastante cercano al río Ojailén, subsidiario del Guadalquivir a través del Jándula. Sin excluir la posible penetración desde la orla atlántica por el Guadiana, creemos que el primer paso debió darse desde Andalucía, en un momento posterior a El Aculadero y a las culturas de las graveras, de las que creemos que son subsidiarias las industrias de cantos tallados de la provincia y que significan el paso siguiente en su evolución.

Después de las series musterienses, limitándonos al área comprendida en nuestras investigaciones, carecemos de testimonios de la presencia humana, excepto la referencia de Obermaier atribuida al Paleolítico Superior que, a tenor de lo investigado por Santonja y Querol, parece que debe ponerse en duda. Por tanto, y mientras no aparezcan otros testimonios, pensamos que el poblamiento debió desaparecer a finales del Würm I y comienzos del II.

A nuestro parecer, las causas de este corte de poblamiento deben buscarse en un hecho antropológico, unido a un aspecto de recursos materiales, y a un factor geofísico. Desde el punto de vista antropológico, hay que situar este despoblamiento en la radical perspectiva de la sustitución foránea del poblamiento existente por el nuevo tipo humano de los Sapiens Sapiens, unido, además, el hecho de la sustitución a la necesidad de búsqueda de otras áreas de recursos materiales más adaptadas a las nuevas técnicas leptolíticas para las que las cuarcitas son muy poco adaptables. En el aspecto geofísico, el progresivo avance de la glaciación Würm supondría en nuestras latitudes una fuerte tendencia a la aridez climática y, en consecuencia, al empobrecimiento general del medio que, unido a la mencionada necesidad de recursos para las industrias, no debieron favorecer la presencia durante la última etapa paleolítica. A nuestro juicio, sería un territorio despoblado durante el Paleolítico Superior hasta los testimonios de un calcolítico temprano de raíces neolíticas finales.

Al finalizar esta visión de síntesis sobre el Paleolítico en Ciudad Real para el justamente merecido librohomenaje al Dr. Vallespí, es para mí un honor y una gozosa obligación dejar constancia de gratitud a la huella que en mí, personalmente, y en la investigación del Paleolítico provincial ha dejado Enrique Vallespí. 
Mi vocación por la investigación paleolítica es fruto del entusiasmo y la dedicación entregada que aprendí de él en los inicios de los años setenta del siglo pasado, como alumno suyo en Prehistoria en la Universidad de Navarra.

Su universal curiosidad científica y las iniciales manifestaciones de mi vocación paleolitista le llevaron a impulsar mis primeros pasos para revisar un yacimiento del que el religioso marianista, Beato Fidel Fuidio, había depositado alguna pieza en el Museo de Vitoria. La positiva verificación y recogida de piezas en Valdarachas, lugar de la localización, afianzaron e impulsaron mis primeros pasos vocacionales y mi relación discente y personal con Enrique Vallespí.

La posterior presencia, como primer Director del Museo de Ciudad Real, del Dr. D. Rafael García Serrano con quien tanto a Enrique como a mí personalmente nos unía una estrecha amistad, también desde los días de la Universidad de Navarra, sirvió de aglutinante para que surgiera un equipo que, con toda la modestia que se quiera, ha marcado un hito en la investigación del Paleolítico provincial.

Nuestra primera actividad de investigación directa se centró en la prospección, en Agosto de 1976, de diversos yacimientos en la cuenca media del Jabalón. Pero indudablemente, el yacimiento estrella fue para nosotros PORZUNA. Su estudio polarizó nuestros trabajos en los veranos de 1978 y 1979. El fruto, la aparición de dos monografías: Achelense y Musteriense de Porzuna (Ciudad Real). Materiales de superficie I (colección E. Oliver) y Achelense y Musteriense de Porzuna (Ciudad Real). Materiales de superficie II (muestra de las colecciones de A. Retamosa y M. Expósito).

Y así, verano tras verano, la E. U. de Magisterio, la Casa Sacerdotal, o el Museo de Ciudad Real, fueron testigos de nuestros desvelos, de nuestra aplicación y del profundo acrisolamiento de nuestra amistad. Más que un equipo, llegamos a ser una familia.

En cuanto a la huella personal que Enrique Vallespí ha marcado en mi actividad de investigador, además de suscitar mi vocación paleolitista, su magisterio se completa con el impulso y dirección de mi tesis doctoral, primera que como investigación sistemática del Paleolítico Inferior y Medio se presentó, bajo su dirección, en la Universidad de Sevilla.

Pero, indudablemente, lo que más me llena de orgullo es la relación personal establecida. Enrique Vallespí supo transcender la mera relación maestro-discípulo por unos derroteros de cordial amistad y de ésta, a una verdadera familiaridad. Desde entonces, Enrique, $\mathrm{M}^{\mathrm{a}}$ Carmen, sus hijos y sus nietos son mi familia de Sevilla, y sus acontecimientos familiares lo han sido también míos.

GRACIAS, Enrique. 


\section{BIBLIOGRAFÍA DE ORIENTACIÓN BÁSICA}

AGUIRREENRÍQUEZ,E. (1971): "Datos para la historia terciaria y cuaternaria del Campo de Calatrava", Cuadernos de Estudios Manchegos, II Época, no 2. Ciudad Real.

CIUDAD SERRANO, A. (1986a): Las industrias de cantos tallados en Ciudad Real. Aportación al Achelense Inferior de la Submeseta meridional, Estudios y Monografías16, Museo de Ciudad Real.

_- (1986b): Las industrias del Achelense Medio y Superior y los complejos musterienses en la provincia de Ciudad Real, Instituto de Estudios Manchegos-Museo de Ciudad Real (Estudios y Monografías, 17). Ciudad Real.

CIUDAD, A.; SERRANO, J. y BARBA, J. (1987): “El Paleolítico del río Tirteafuera”, Oretum III: 3-38.

HERNÁNDEZ PACHECO, E. (1921): "El yacimiento de mamíferos cuaternarios de Valverde de Calatrava y edad de los volcanes de Ciudad Real", Mem. R. Soc. Esp. His. Nat.: 98-114. T. cincuentenario, Madrid.

MOLINA, E. (1972): "Observaciones geológicas en el Campo de Calatrava”, Estudios Geológicos XXVIII: 3-11. Madrid.

MONTURIOL, F.; GALLARDO, S. y ALEIXANDRE, T. (1970): “Los paleosuelos rojos en las formaciones cuaternarias del Campo de Calatrava", Anales de Edafología y Agrobiología XXIX (7-8): 549-573. Madrid.

REDONDO, E. y MOLINA, E. (1980): "Bosquejo morfológico de la cuenca del río Bul1aque (Ciudad Real)", Bol. del Inst. Geológico y Minero, XCI (Tercer fasc., Mayo-Junio): 30-40, Madrid.

SANTONJA GÓMEZ, M. (1981): "Características generales del Paleolítico inferior de la Meseta Española”, Numantia: 9-64. Soria.

VALLESPÍ, E.; CIUDAD, A. y Ga. SERRANO, R. (1979): Achelense Musteriense de Porzuna (Ciudad Real). Materiales de superficie I. (Col. E. Oliver), Estudios y monografías 1. Museo de Ciudad Real (1985): Achelense Musteriense de Porzuna (Ciudad Real). Materiales de superficie II. (Muestra de las Colecciones de A. Retamosa, y M. Expósito) Universidad de Castilla-La Mancha, Ciudad Real. 